\title{
A COVID-19 Outbreak - Nangong City, Hebei Province, China, January 2021
}

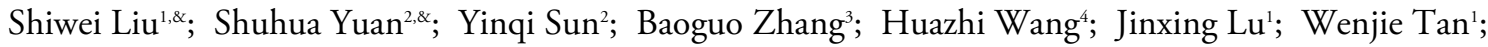 \\ Xiaoqiu Liu'; Qi Zhang'; Yunting Xia ${ }^{1}$; Xifang Lyu' ${ }^{1}$; Jianguo $\mathrm{Li}^{2, *}$; Yan Guo ${ }^{1, *}$
}

\section{Summary \\ What is known about this topic?}

Coronavirus disease 2019 (COVID-19) is widespread globally. In China, COVID-19 has been well controlled and has appeared only in importationrelated cases. Local epidemics occur sporadically in China and have been contained relatively quickly.

What is added by this report?

Epidemiological investigation with genome sequence traceability analysis showed that the first case of COVID-19 in Nangong City acquired infection from a confirmed case from Shijiazhuang City; infection subsequently led to 76 local cases. All cases were associated with the index case, and most were located in Fenggong Street and did not spread outside of Nangong City. The main routes of transmission were family clusters, intra-unit transmission, and nosocomial transmission.

What are the implications for public health practice?

This study highlights new techniques for rapidly tracing cases and identifying COVID-19 transmission chains. The different epidemiological characteristics in Nangong City, from the earliest stages of the outbreak, suggest that allocation of health sources for prevention and treatment were reasonable. Preventing transmission within medical institutions and isolation facilities and strengthening management in the community should be priorities for COVID-19 control during a city lockdown.

Coronavirus disease 2019 (COVID-19) is an infectious disease caused by a novel coronavirus (also known as severe acute respiratory syndrome coronavirus 2; SARS-CoV-2) that was declared a pandemic by the World Health Organization (WHO) in March 2020. COVID-19 is widespread globally but has been well controlled in China with only scattered, importation-related local outbreaks following containment in April 2020.
On January 3, 2021, Nangong City (part of Xingtai City, Hebei Province with 484,000 residents in 2017) reported its first symptomatic case of COVID-19. China CDC, Hebei CDC, and Xingtai CDC jointly carried out a field epidemiological investigation and traced the outbreak. On January 6, Nangong City started its first round of population-wide nucleic acid screening. On January 9, Nangong City was locked down by conducting control and prevention measures that included staying at home and closing work units for seven days. As of January 27, 2021, 76 cases had been reported in Nangong City; among these, 8 were asymptomatic. No additional cases have been reported to date, and there were no COVID-19-related deaths in the outbreak.

\section{INVESTIGATION AND FINDINGS}

The index COVID-19 case in Nangong City is a 34-year-old male office worker (Patient A) when he noticed a fever and took his temperature, finding it to be $38.1{ }^{\circ} \mathrm{C}$ at $14: 00$ on January 1,2021 . At $15: 30$, he drove to the fever clinic of Nangong People's Hospital for evaluation and treatment and was screened for COVID-19 by nucleic acid testing. Patient A was informed on January 2 that his nucleic acid test was positive. On January 3, Patient A was diagnosed as a confirmed case of symptomatic COVID-19 and treated in isolation.

The field epidemiological investigation and case tracing found that Patient A went to the respiratory department of Hebei Children's Hospital in Shijiazhuang City on December 25, 2020 and had close contact with a symptomatic case of COVID-19 in a waiting area. The contact, Patient $\mathrm{B}$, female, from Gaocheng District, Shijiazhuang City, had asthma and was febrile on December 25 (highest temperature $38.5^{\circ} \mathrm{C}$ ). Video monitoring showed Patient A and B were in a room 4.5 meters apart from each other for 16 minutes. During this period, they did not talk or touch the same objects. Patient B removed her face mask 
twice to eat, and Patient A pulled down his face mask to make phone calls. Whole genome sequence traceability analysis from Hebei CDC found that the viral nucleic acid sample from Patient $A$, compared with the Wuhan reference strain (NC_045512), had mutation characteristics of the gene locus of the European family of the L genotype and was on the B.1.1.123 branch. The genome sequence has 21 nucleotide mutations that are same as virus samples from Gaocheng District cases. The genetic sequences of Patient A's and Patient B's viruses were highly homologous, presumably representing a common transmission source.

Considering dates of illness onset identified by the field epidemiological investigation and the sampling date of the first positive nucleic acid test, the first COVID-19 infection in Nangong City occurred on January 1 . Since then, the number of cases reported each day increased rapidly and peaked on January 13 with 12 cases reported. Following the peak, the number of cases reported each day decreased until the last case was reported on January 23. The cumulative number of cases increased fastest from January 9 to January 13 (Figure 1). Among all 76 cases, 46 (60.5\%) were among males; 63 (82.9\%) were among young to middle-aged (15-59 years old) individuals; the ages ranged from 2 to 83 years with a median of 34.5 years; $34(44.7 \%)$ were farmers, $11(14.5 \%)$ were office staff, and $10(13.2 \%)$ were health care workers; $59(77.6 \%)$ came from Fenggang Street, and 17 (22.4\%) came from seven other streets or townships (there are 15 streets and townships in Nangong City) (Figure 2, Table 1). Symptoms among the 68 symptomatic cases were generally moderate or mild.

Two cases, including the index case, were identified by fever clinics; 16 were found by community-wide nucleic acid screening; 5 were detected by daily routine tests for medical staff conducted by hospitals; and 53 were discovered by regular, confirmatory nucleic acid screening of close contacts or sub-close contacts including 48 (63.2\%) identified during centralized isolation or quarantine.

Transmission chains were characterized by mixed existence of intra-unit transmission, family clusters, nosocomial infections, and community transmission associated with the index case Patient A. There was 1 intra-unit transmission with $23(30.3 \%)$ people infected, 2 family clusters (identified by $\geq 5$ people infected) with $17(22.4 \%)$ people infected, 3 retransmissions in medical institutions with $8(10.5 \%)$ people infected, and 1 community cluster (identified by $\geq 5$ people infected) with $6(7.9 \%)$ people infected. There was the possibility of cross-infection in isolation places, but there is no definitive supporting evidence. All COVID-19 cases in Nangong City were related to the index case, Patient A; eight generations of infection were observed in the transmission chains.

\section{DISCUSSION}

This was a local outbreak of COVID-19, with the first case coming from a known epidemic area (Gaocheng District, Shijiazhuang City). The epidemic was confined to a limited area - mainly Fenggang Street — and did not spread outside Nangong City, indicating that the strategies used in Nangong City

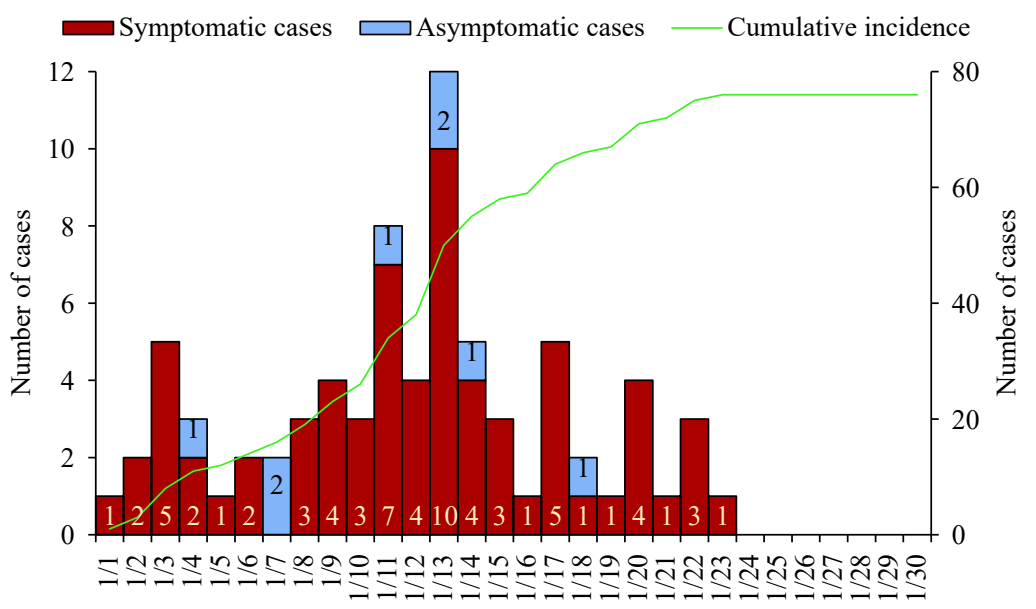

Date of illness onset

FIGURE 1. Distribution of date of illness onset based on field epidemiological investigation and cumulative incidence of COVID-19 cases from January 1 to January 23, 2021 in Nangong City, Hebei Province, China. 


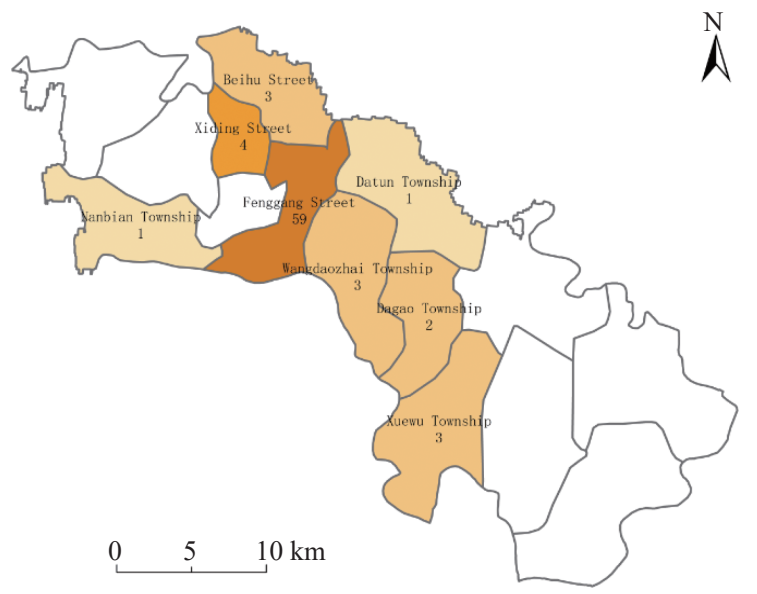

FIGURE 2. Geographic distribution of the 76 COVID-19 cases reported in Nangong City, Hebei Province, China, January 3 to January 27, 2021.

were highly effective.

Compared with the earliest cases in China during 2019-2020 (1-2), the epidemic in Nangong City was characterized by milder symptoms and faster and more insidious transmission and occurred mainly young and middle-aged adults. These characteristics suggest reasonable allocation of health sources and effective prioritization of health policies in the control, prevention, and treatment of COVID-19.

Application of new technologies and new investigation methods were crucial for case tracing and identification of transmission chains. For various reasons, it can sometimes be difficult to provide exact information in field epidemiological investigation reports. When possible, we should integrate digital techniques, big data, trajectory tracking systems, monitoring videos, and sensitive and timely laboratory detection technologies into field investigations. Indeed, in the Nangong City outbreak, source identification for the first case and transmission chain identification for subsequent cases were accomplished with the assistance of monitoring video surveys.

Early and proactive measures are highly effective and

TABLE 1. Epidemiological characteristics of the 76 COVID-19 cases reported in Nangong City, Hebei Province, China, January 3 to January 27, 2021.

\begin{tabular}{|c|c|c|c|c|c|c|}
\hline \multirow{2}{*}{ Characteristics } & \multicolumn{2}{|c|}{ Symptomatic cases } & \multicolumn{2}{|c|}{ Asymptomatic cases } & \multicolumn{2}{|r|}{ Total } \\
\hline & $\mathbf{N}$ & Percentage (\%) & $\mathbf{N}$ & Percentage (\%) & $\mathbf{N}$ & Percentage (\%) \\
\hline Total & 68 & 89.5 & 8 & 10.5 & 76 & 100.0 \\
\hline \multicolumn{7}{|l|}{ Gender } \\
\hline Female & 29 & 42.6 & 1 & 12.5 & 30 & 39.5 \\
\hline Male & 39 & 57.4 & 7 & 87.5 & 46 & 60.5 \\
\hline \multicolumn{7}{|l|}{ Age group (years) } \\
\hline$<15$ & 5 & 7.4 & 1 & 12.5 & 6 & 7.9 \\
\hline $15-29$ & 17 & 25.0 & 2 & 25.0 & 19 & 25.0 \\
\hline $30-44$ & 20 & 29.4 & 3 & 37.5 & 23 & 30.3 \\
\hline $45-59$ & 19 & 27.9 & 2 & 25.0 & 21 & 27.6 \\
\hline$\geq 60$ & 7 & 10.3 & 0 & 0 & 7 & 9.2 \\
\hline \multicolumn{7}{|l|}{ Occupation } \\
\hline Farmer & 31 & 45.6 & 3 & 37.5 & 34 & 44.7 \\
\hline Office staff & 11 & 16.2 & 0 & 0 & 11 & 14.5 \\
\hline Health care worker & 8 & 11.8 & 2 & 25.0 & 10 & 13.2 \\
\hline Workman & 5 & 7.4 & 1 & 12.5 & 6 & 7.9 \\
\hline Teacher & 5 & 7.4 & 0 & 0 & 5 & 6.6 \\
\hline Student & 4 & 5.9 & 1 & 12.5 & 5 & 6.6 \\
\hline Child & 2 & 2.9 & 1 & 12.5 & 3 & 3.9 \\
\hline Business services & 2 & 2.9 & 0 & 0 & 2 & 2.6 \\
\hline \multicolumn{7}{|l|}{ Location } \\
\hline Fenggang Street & 52 & 76.5 & 7 & 87.5 & 59 & 77.6 \\
\hline Other streets & 16 & 23.5 & 1 & 12.5 & 17 & 22.4 \\
\hline
\end{tabular}


play an important role in rapid control of infectious diseases (3) as was seen in the COVID-19 outbreak in Nangong City. First cases are usually identified in medical institutions, suggesting the necessity of continuously improving the sensitivities of symptom surveillance, fever screening, and disease diagnosis in hospitals, private clinics, and even pharmacies. We should therefore strengthen and routinize standardized construction and management of fever clinics and their training of professionals. We should work to avoid nosocomial infection of COVID-19, and we should carry out rapid and accurate risk assessments in outbreaks, using nucleic acid testing for close contacts and people at high risk to provide evidence to further improve evaluation and implementation of measures.

When vaccines and effective medicines are not available on-demand, strictly implementing containment and suppression public health strategies (4) is urgently needed once an outbreak happens. However, it is challenging to prevent transmission in community and isolation points under a city lockdown. For communities, in addition to implementation of relevant control measures, we should ensure supplies for normal living, strengthen management of service providers and volunteers, and promote health education. In isolation points, we should take strict prevention measures, such as staying in separate rooms, not allowing visitors, ensuring adequate ventilation, employing sensitive fever screening, and properly disinfecting the environment.

Personal protection is one of the most effective ways to prevent infection. Measures should include maintaining social distance, hand hygiene, respiratory etiquette, face mask use, and not smoking. In the Nangong City epidemic, the index case, Patient A, was likely infected after he pulled down his face mask to make a phone call. Case number 75 was likely infected when removing his or her face mask for smoking while in the presence of a COVID-19 carrier.

Acknowledgements: Hong Jin, Xiaofeng Zhang, Yonggui Du, Yujie Cui, Siqi Zhang, Ying Wang, Junqin Zhao, and Lin Ma from Hebei CDC; and Xianghong Meng and Xuejie Gao from Xingtai CDC.

doi: $10.46234 / \mathrm{ccdcw} 2021.077$

\# Corresponding authors: Jianguo Li, hblpss13@163.com; Yan Guo, guoyan@chinacdc.cn.

\footnotetext{
${ }^{1}$ Chinese Center for Disease Control and Prevention, Beijing, China;

2 Hebei Provincial Center for Disease Control and Prevention, Shijiazhuang, Hebei, China; ${ }^{3}$ Xingtai Center for Disease Control and Prevention, Xingtai, Hebei, China; ${ }^{4}$ Nangong Center for Disease Control and Prevention, Nangong, Hebei, China.

$\&$ Joint first authors.
}

Submitted: March 12, 2021; Accepted: March 18, 2021

\section{REFERENCES}

1. The Novel Coronavirus Pneumonia Emergency Response Epidemiology Team. The epidemiological characteristics of an outbreak of 2019 novel coronavirus diseases (COVID-19)—China, 2020. China CDC Wkly 2020;2(8):113 - 22. http://dx.doi.org/10.46234/ccdcw2020.032.

2. Wu ZY, McGoogan JM. Characteristics of and important lessons from the coronavirus disease 2019 (COVID-19) outbreak in China: Summary of a report of 72314 cases from the Chinese Center for Disease Control and Prevention. JAMA 2020;323(13):1239 - 42. http://dx.doi.org/10. 1001/jama.2020.2648.

3. Zhou L, Wu ZY, Li ZJ, Zhang YP, McGoogan JM, Li Q, et al. One hundred days of coronavirus disease 2019 prevention and control in China. Clin Infect Dis 2021;72(2):332 - 9. http://dx.doi.org/10.1093/ $\mathrm{cid} / \mathrm{ciaa} 725$.

4. Li ZJ, Chen QL, Feng LZ, Rodewald L, Xia YY, Yu HL, et al. Active case finding with case management: the key to tackling the COVID-19 pandemic. Lancet 2020;396(10243):63 - 70. http://dx.doi.org/10.1016/ S0140-6736(20)31278-2. 